Article original

\title{
Mort périnatale et d'un jeune enfant. Histoire des rites et des pratiques funéraires en Europe issus de l'expression affective et sociale du deuil. Première partie : de la Préhistoire aux Lumières
}

\section{Stillborn children and infant death. History of funeral practices and rites in Europe stem from affective and social expression of grief. First chapter: From Prehistory to Enlightenment}

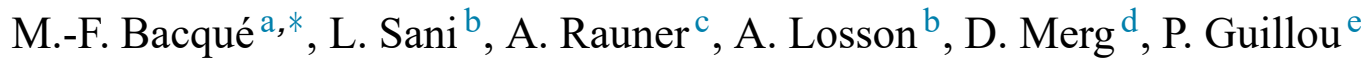 \\ ${ }^{a}$ EA 3071, SuLiSoM, société de thanatologie, université de Strasbourg, faculté de psychologie, 12, rue Goethe, 67000 Strasbourg, France \\ ${ }^{\mathrm{b}}$ EA 3071, SuLiSoM, université de Strasbourg, faculté de psychologie, 67000 Strasbourg, France \\ ${ }^{c}$ EA 3440 ARCHE, université de Strasbourg, faculté d'histoire, 67000 Strasbourg, France \\ ${ }^{\mathrm{d}}$ Hôpitaux universitaires de Strasbourg, pôle Mère-Enfant, Association « Nos tout-Petits d'Alsace », hôpital de Hautepierre, 67200 Strasbourg, France

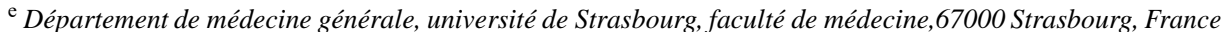

\begin{abstract}
Résumé
But de l'article. - Cette recherche interdisciplinaire est consacrée à l'évolution historique des pratiques funéraires en réponse aux demandes émotionnelles des parents en deuil d'un jeune enfant à partir de la naissance.

Méthodes. - Revue des publications sur l'histoire des pratiques et les rites funéraires des jeunes enfants.

Résultats. - On trouve des pratiques funéraires pour les enfants dès la Préhistoire. L'Antiquité est riche en monuments, épitaphes, objets funéraires réservés aux tout-petits. Ils signent la puissance économique ou politique des familles et montrent l'intensité de la souffrance des parents. Regret et nostalgie expriment la tristesse que l'enfant n'ait pu réaliser sa promesse de vie. Pour autant, les funérailles restent discrètes pour les enfants morts avant la pousse des dents. Malgré les infanticides qui semblent ne pas donner lieu à culpabilité ou à deuil, on ne peut pas conclure à l'indifférence des parents. Le Moyen Âge en Europe est surtout marqué par l'hégémonie de l'Église catholique qui refuse le repos de l'âme de l'enfant non baptisé. Le Limbus puerorum dans lequel errent les âmes ne satisfait pas les parents qui cherchent à tout prix le salut de leur enfant. Une possibilité de rédemption réside néanmoins dans les sanctuaires à répit.

Conclusion. - La souffrance des parents est certaine dans toutes les périodes préhistoriques et historiques même si la mortalité élevée pourrait banaliser la mort des enfants. Elle s'exprime via les pratiques funéraires et la question de l'accès à l'au-delà, en raison de l'importance de la religion, ciment des sociétés antique et médiévale. Les rites sociaux sont recherchés pour leur soutien moral et leur inscription dans la société.
\end{abstract}

Mots clés : Rites et pratiques funéraires ; Enfants morts à partir de la naissance ; Deuil ; Représentations de la mort ; Famille au Moyen-Âge

Abstract

Aim. - Interdisciplinary research of emotional constants and the need for social support in grieving parents throughout the ages.

Methods. - State of the arts of historical publications from 1970 to 2017 on funeral rites for stillborn and young children.

Findings. - There is some evidence of parents suffering through funeral rites for children in Prehistory, even for a foetus. Since Antiquity, many monuments, epitaphs and funeral furniture have been found for children. Although this is evidence of an economic and political power of patrician families, it is also evidence of parents' grief suffering. If many infanticides do not seem to have been followed by guilt or grief, the funeral rites for the natural death of a predental child appear to have been very discreet. Parents were sensitive to the death of their child. This is confirmed for

\footnotetext{
* Auteur correspondant.

Adresse e-mail : mfbacque@club-internet.fr (M.-F. Bacqué).
} 
rich families but could not be proven for slaves or even the poor because of a lack of evidence. The Middle Ages in Europe are characterized by the hegemony of the Catholic Church which refused to baptize dead people. Stillborn children represented a problem for families because if they were not baptized by priests or midwives, their soul would stay in Hell for eternity. To reassure parents, the Catholic Church created a space between its two central places in its spiritual geography, Hell and Heaven. The Limbus puerorum was a virtual space for unbaptized children, but this was unsatisfactory for Middle Ages' parents. With the creation of Purgatory, a place for redemption was possible for sinners but not for unbaptized children. The Catholic Church was literally compelled to accept these opportunities because of complaints from families whose suffering was double: parents had not only lost a child but this child would be doomed for eternity. Stillborn children have not kept out sins despite their short life as edited by Saint Augustin (5th century). The parents felt responsible and guilty for this shameful spiritual status. Redemption of sins could be obtained through "sanctuaires à répit", special places where a child could have "rebirth" and be baptized until he died once again. Conclusion

Parents' grief can be proven throughout all historic and even prehistoric times. Despite high child mortality rates, the death of a child was not a reality easily admitted by families. Social funeral rites were followed by parents for their moral support, but Catholic Church hegemony in Europe of the Middles Ages became controversial and especially with the development of Protestantism. Until the Enlightenment, the salvation of the soul of a stillborn child constituted a real stake for families. This situation was well understood by Catholic Church which used it to enlarge the baptized community as well maintain power on mourning families as well as tranquilizing parents who had lost a baptized child.

Keywords: Funeral rites; Stillborn children; Infant death; Grief; Death representations; Middle-Ages mourned family

\section{Introduction}

La mort d'un jeune enfant a toujours fait l'objet de soins particuliers. Ils témoignent de l'intérêt des parents pour leur progéniture, autant dans un projet de descendance que dans la réalisation d'un attachement émotionnel. Cet attachement à l'enfant, au cours des différentes périodes historiques, est perceptible sous des traces difficiles à interpréter et même à comprendre, des centaines d'années plus tard. Le thème général de cet article porte sur les pratiques de deuil des parents d'un jeune enfant mort, dans les sources les plus anciennes jusqu'à aujourd'hui. Notre expérience actuelle avec ces parents démunis et les difficultés des soignants à proposer des réponses autres que strictement thérapeutiques, montrent un écart entre les réactions affectives et sociales à la perte d'un nouveau-né dans un contexte médicalisé et le besoin de symbolisation ancestral du passage de la vie à la mort [1].

Les rites forment un ensemble défini de cérémonies et de pratiques sociales qui marquent et accompagnent les changements d'état de l'être humain. Les rites de passage comprennent principalement les rites de naissance, de puberté, d'alliance et les rites de mort [2]. Invariablement, la force et l'efficacité d'un rite funéraire consistent à évoquer trois niveaux :

- le niveau symbolique du cycle de la vie et de la résistance à la mort ;

- le niveau social de la désorganisation du groupe qui va retrouver son équilibre dans la répétition de coutumes et usages reconnus;

- le niveau spirituel qui rappelle la mythologie de la genèse de la communauté, formée d'êtres uniques et universels [3].

Plus concrètement, les rites funéraires permettent l'expression et la légitimation des émotions extrêmes dues à la mort tout en les normalisant par la présence du groupe (souvent des témoins expérimentés) et en rétablissant le désordre par l'assurance de la pérennité de la société. Pourquoi alors, malgré leur efficacité, les rites se sont-ils progressivement affaiblis dans les sociétés modernes ? Les aspects biologiques des passages de l'existence (naissance, puberté, procréation et mort) se sont lentement dégagés de la tutelle religieuse pour trouver une légitimité médicale. De plus, la laïcisation de la société française, propose peu de pratiques rituelles lors de la perte d'un nouveau-né.

Devant la souffrance des parents, est-il possible de revivifier les pratiques rituelles d'accompagnement lors de la mort d'un enfant par l'intermédiaire des soignants ou d'autres praticiens?

Notre revue de la littérature et nos études préliminaires font apparaître un problème général de recherche : les rites et pratiques funéraires sont progressivement transférés sur les fonctions thérapeutiques des professionnels de santé qui s'estiment majoritairement non formés [4].

Les rites funéraires existent depuis la Préhistoire [5]. À partir de ces sources préhistoriques et historiques, nous allons déterminer les caractéristiques des pratiques reposant sur des contenants (tombes), des signes (masques mortuaires, inscriptions, vêtements, amulettes), des lieux sacrés et des discours rapportés dans des écrits et des objets artistiques.

L'évolution des représentations de l'enfant produit de nouvelles façons d'évoquer sa mort avec les portraits peints et photographiés du $\mathrm{XIX}^{\mathrm{e}}$ siècle. Le $\mathrm{XXI}^{\mathrm{e}}$ siècle marque un changement avec les photos et les vidéos transmises aux réseaux sociaux.

Mais l'évolution de toutes ces pratiques ne repose pas seulement sur les vestiges mis au jour. Il faut interpréter les sources historiques qui ne permettent pas de retrouver directement les gestuelles, les comportements et encore moins les émotions collectives et individuelles éprouvées, du moins pour les périodes les plus anciennes.

Notre question de recherche spécifique se situe donc au carrefour de différentes approches, historique, socio-anthropologique et psychologique. Comment les parents ont-ils historiquement eu recours aux croyances, aux religions, puis au droit et à la médecine pour accepter la rupture de leurs liens avec un enfant 
en gestation ou très jeune? Que reste-t-il aujourd'hui de ces pratiques et rituels dans l'accompagnement de ce deuil complexe?

Si les soignants (médecins, sages-femmes, infirmiers, psychologues) sont sensibles à l'attachement parental, il leur paraît difficile aujourd'hui d'introduire les rites ancestraux du deuil. Le droit a tenté de poser des limites pour l'inscription sociale d'un enfant dans la société. La médecine cherche aussi à trouver les moyens de pallier les émotions tristes, voire la psychopathologie du deuil des parents.

L'équipe à l'origine de ce travail est composée de praticiens auprès de parents endeuillés et d'une spécialiste de la méthodologie des sciences historiques. Nous avons ainsi pu poser la question interdisciplinaire de l'existence d'invariants affectifs et sociaux après une mort périnatale ou d'un jeune enfant, ainsi que de la constance de la demande de soutien des endeuillés à la communauté sociale.

\section{Méthodologie}

Il s'agit d'une revue de la littérature internationale portant sur les rituels funéraires européens dans le cas de la mort périnatale et de celle du jeune enfant.

Nous avons procédé à la lecture de tous les articles depuis 1970 sur les bases de données accessibles. PubMed propose 11899 articles contenant le mot clé Perinatal death, mais seulement 19 articles lorsqu'on croise ce mot-clé avec le mot clé Funeral. Treize articles sont proposés sur PsycInfo lorsque l'on croise Death and Dying, Emotional et Perinatal death. Ces derniers traitent essentiellement des effets psychologiques de la perte d'un enfant.

La base Cairn contient beaucoup plus d'articles, et de références d'ouvrages francophones. La France s'est particulièrement illustrée par ses travaux historiques, avec Philippe Ariès et Michel Vovelle, Anne-Marie Tillier, Danièle AlexandreBidon, Cécile Treffort, Régis Bertrand.

À partir de notre thématique générale, nous avons tenté de mieux comprendre l'évolution des rites et des émotions exprimées par les parents endeuillés jusqu'à nos jours afin de répondre à nos questions de recherche. Cantonnés aux pratiques et rituels chrétiens, majoritaires en Europe, nous sommes conscients que les pratiques juives et musulmanes doivent également être recensées, mais ceci fera l'objet d'un autre article.

Nos résultats comprennent donc une synthèse des rapports archéologiques, des extraits de témoignages ou d'épitaphes, des articles historiques, sociologiques, anthropologiques et ou psychologiques, pour inclure même des sites internet récents qui présentent aussi des hommages aux enfants disparus, des expressions émotionnelles langagières ou imagées grâce à des photos et des films qui nous permettront de tracer une frise de l'évolution des rites funéraires dédiés aux tout jeunes enfants disparus (disponible dans le second chapitre de cette recherche qui est publiée en deux temps).

Le problème de l'âge des enfants n'a pas été résolu. Alors que nous ne souhaitions travailler qu'avec des tout-petits, de la naissance jusqu'à l'âge d'un an, la plupart des documents archéologiques et historiques ne sont pas aussi précis qu'espéré au début de l'étude. Les différents statuts de l'enfant (pousse des dents, marche) seront donc précisés lorsqu'ils entraînent des différences dans le deuil.

Enfin, dernier point et non des moindres : les sciences historiques ne traitent que depuis très peu de temps de la question de la mort des tout-petits. L'histoire de la mort est elle-même une histoire récente, qui émerge dans les années 1960, alors que l'archéologie s'en est emparée depuis plus longtemps. Nous retrouvons des articles sur les funérailles des enfants dans le cadre de l'étude des tombes familiales dès 1970-1980, cependant, seules les années 2000 marquent les débuts de l'intérêt des historiens pour ce sujet. Rappelons finalement que les sources de l'historien ont des limites qui empêchent de percevoir tous les aspects du deuil du jeune enfant. Évidemment, certaines d'entre elles ont totalement disparu. De fait, les populations les plus aisées ont laissé plus de traces (archéologiques, iconographiques, textuelles) que les autres. Dans ces conditions, il est difficile de percevoir les réalités du deuil chez les pauvres, les esclaves, les exclus. Mais surtout, l'historien est confronté à la question de l'expression des émotions qui n'est pas identique selon les époques [6] ainsi qu'à la place plus ou moins grande de l'enfant dans les différentes sociétés et donc dans les sources.

C'est donc avec toutes ces restrictions que nous pouvons débuter cette étude interdisciplinaire des témoignages de pratiques qui transcendent les émotions vécues depuis des millénaires. Notre premier résultat est qu'une forme constante d'attachement aux enfants a constitué pendant longtemps le seul espoir de prolongation et de dépassement d'un individu et de sa lignée.

\section{La Préhistoire}

Les preuves attestées de rites funéraires préhistoriques remontent au moins à 100000 ans, et celles comportant des restes d'enfants, voire de fœtus ne sont pas si rares. Mentionnons la grotte de La Ferrassie en Périgord [5]. Elle a livré les restes de sept Néanderthaliens (datés entre -75000 et -60000 ans), deux adultes, trois enfants d'environ deux, trois et dix ans, un nourrisson et un fœtus de sept mois [7]. Le fœtus est accompagné de beaux outils de silex, ce qui montre que sa mort ne laisse pas indifférents les proches qui ont réalisé la sépulture [8]. Il n'y a cependant pas de lieu de sépultures spécifiques pour ces tout-petits qui sont inhumés avec les autres défunts, à quelques rares exceptions près [9].

\section{L'Antiquité}

Laissons la Préhistoire pour nous attacher à l'Antiquité européenne à partir de 3500 ans av. J.-C. jusqu'en 476 ap. J.-C.

Les enfants morts sont pratiquement toujours inhumés [10].

De nombreuses recherches montrent l'importance des tombes d'enfant [11], mais aussi la douleur des parents et des proches, même si ce n'est qu'à demi-mots ou de manière indirecte.

L'Âge de Bronze, à partir de 3000 av. J.-C. permet de trouver dans les cités de nombreux lieux d'inhumation des jeunes enfants. Les enfants sont ensevelis dans les espaces habités des 
maisons (cuisine, salle de réception, espace central, escaliers ou près de l'entrée).

Les petits corps sont placés dans des récipients en céramique, comme des marmites, de grands bols ou des bassins [12,13]. Les différentes inscriptions funéraires n'évoquent pas la personne de l'enfant mais plutôt sa personnalité sociale, principalement son sexe et sa place dans la lignée.

Les restes d'enfants sans statut social ne sont pas retrouvés et limitent nos interprétations. En Grèce, les tombes d'enfant comportent souvent du mobilier funéraire. La mort de l'enfant ne semble pas troubler plus que celle des grands, mais ils bénéficient de célébrations et de commémorations. Le deuil est institutionnalisé et les émotions intimes du chagrin sont discrètes derrière des cérémonies funéraires déjà très codifiées. Les femmes sont associées au débordement et les hommes à la mâ̂trise. Pour tous les défunts en effet, l'expression publique et privée du deuil se répartit selon le genre : aux femmes (et aux esclaves) la partie privée du deuil et les soins au cadavre entraînant potentiellement la souillure de la mort, aux hommes (les citoyens), la cérémonie publique renforçant la cohésion sociale et la transmission de l'histoire commune [14].

\section{Le monde romain (753 av $\mathrm{J}-\mathrm{C}-476$ après $\mathrm{J}-\mathrm{C}$ )}

L'ensevelissement systématique des morts est justifié par la crainte de leur transformation en larvae, ces morts malfaisants qui n'ont pas franchi le Styx [15]. Les larvae sont des âmes errantes et nuisibles. Elles sont issues soit d'une mort violente, soit d'une mort prématurée, soit n'ont pas eu de sépulture rituelle (absence ou violation de sépulture, abandon des sacra privata). Les enfants morts en bas âge sont donc tout sauf des quiescentes animae. C'est pourquoi les magiciens placent dans leur tombeau des tablettes de plomb. Les larvae peuvent par ailleurs avoir la puissance d'un guérisseur [16]. L'enfant mort en bas âge et surtout avant le don du cognomen (surnom) par son père au $9^{\mathrm{e}}$ jour, est considéré comme n'étant pas entré de façon complète dans la société des vivants. La tristesse retrouvée sur les épitaphes témoignerait ainsi de leur regret de la vie. Cependant, si l'enfant est intégré par les rites de naissance (comme le premier bain par exemple), sa mort ne le conduit pas à devenir un être malfaisant [17].

À partir du règne d'Auguste (28 av. J.-C.- 14 ap. J.-C.) jusqu'à l'époque de Marc-Aurèle (161-180 ap. J.-C), tous les enfants légitimes de citoyens romains doivent être inscrits sur le registre des naissances dans les trente jours qui suivent leur mise au monde, et seulement après l'attribution de leur cognomen [13].

Des comportements et des rites spécifiques accompagnent la mort des enfants. Tombes et épitaphes expriment, paradoxalement de façon pérenne, les limites d'une vie caractérisée par sa fragilité. D'une part, elles avertissent les femmes et les hommes de la cruauté implacable de la vie et inscrivent les voix des enfants dans la pierre ou l'argile pour toujours. D'autre part, elles montrent la puissance de ces familles capables de commémorer leur progéniture disparue [12].

Dans tout l'Empire, les inscriptions funéraires ont une valeur familiale, mais surtout sociale, par les strictes informations jugées nécessaires et appropriées aux circonstances. Les démonstrations émotionnelles du deuil sont considérées comme déplacées ou de mauvais goût (les pleureuses se substituent aux parents). L'âge du défunt détermine la durée du deuil : plus l'enfant a vécu, plus la démonstration du chagrin est marquée. « On ne portait pas le deuil d'un enfant en dessous de trois ans, et pour tous ceux qui avaient dépassé cet âge, on ne le portait pas pendant plus de mois qu'ils n'avaient vécu d'années, sans dépasser dix mois, quel que fût l'âge du défunt », explique Plutarque (Vie de Numa, 12.3, cité par Dasen [18], p. 2). Les tout-petits, morts avant la pousse des dents, sont inhumés parfois dans le sol de l'habitation, plus souvent aux alentours. Leurs funérailles sont rapides, nocturnes, sans longue exposition du corps à la maison, sans pleureuses, ni oraison publique. Les hommes ne se manifestent pas comme les femmes qui ont une forme codifiée de «permission » sociale. On trouve déjà l'inscription nolite dolere, parentes (mes parents, ne souffrez pas) et cet appel du petit défunt : «Quelle joie, pour les miens, que mon premier anniversaire ! Mais ravi par un Sort cruel, point n'ai-je pu en fêter d'autre. Un petit bout d'année (annuclus), sept mois, et le souffle me manque. Arraché à leurs bras, je flotte maintenant, parmi les ombres vaines. Pourquoi griffer ton ventre et frapper ta poitrine, ô maman ? Pas un mortel ne peut se garder de la mort » (Tombe de Cn. Domitius Proculus, Rome CIL VI 35126 ; trad. Porte 1993, p. 52-53, art. cit. p. 4). L'inscription dans la pierre pérennise sans doute l'amour des endeuillés pour leurs enfants mais elle a aussi pour fonction de prévenir les autres parents des risques de l'attachement tandis qu'elle normalise le deuil dans l'espace public.

Il y a plus de commémorations pour les garçons que pour les filles : dans la société romaine l'homme occupe une position dominante par rapport à la femme. Cependant, la perte d'une petite fille peut aussi être célébrée parce qu'elle éveille d'abord tendresse et douceur (les femmes transmettent aussi la citoyenneté) alors que la perte d'un garçon entraîne le deuil d'un futur citoyen au rôle reconnu dans la cité. Ainsi, un autel funéraire $\mathrm{du} \mathrm{II}^{\mathrm{e}}$ siècle avant notre ère représente deux très jeunes enfants morts, l'un à onze mois et huit jours, l'autre à un an, cinq mois et dix jours, comme deux écoliers studieux, debout en toge, tenant dans la main un rouleau de papyrus. Quand une inscription donne l'âge réel de l'enfant, son portrait le représente plus âgé, comme pour montrer ce qu'il aurait dû devenir et proroger virtuellement son existence terrestre. Lisons l'épitaphe trouvée à Mayence : «Voici qu'il faut pleurer une très douce petite fille, (puellula). Il aurait mieux valu ne pas venir au monde, si, toi, qui serais devenue si charmante, tu avais pour destinée, dès ta naissance, de retourner vite d'où tu étais venue et d'être une cause de deuil pour tes parents. Elle a vécu six mois et huit jours. Rose, elle a fleuri et péri aussitôt » (trad. Néraudau 1984, p. 380-381; CIL XIII 7113, in Dasen [18], art. cit. p. 4).

La plupart des poèmes trouvés en épitaphe ont des thèmes récurrents : la cruauté du sort et la précarité de la vie [19].

Les hommes ne sont pas autorisés à pleurer la mort de leur fils, ni à être absents du travail. Mais pour les femmes, le deuil dure de neuf à dix mois au maximum pour la perte d'un père, d'un fils ou d'un frère. 
Pourtant, à l'époque impériale, les chagrins, même paternels sont autorisés par la société mais sans manifestation publique bruyante. Les femmes peuvent se livrer à la lamentation : « Ne cherche pas à t'excuser sur la faiblesse de ton sexe, écrit Sénèque à sa mère Helvia, c'est presque un droit qu'on lui accorde de pleurer avec excès ». Le législateur leur impose néanmoins des limites, « afin de composer, par une règle officielle, avec l'obstination de la douleur des femmes. Ils n'ont pas interdit le chagrin, mais ils l'ont borné » (Consolation à ma mère Helvia, 16.1, in Dasen [18], art. cit. p. 3).

Les sociétés humaines ont toujours éprouvé de l'horreur pour le processus de décomposition, horreur aujourd'hui transformée en crainte. C'est pourquoi le cadavre, après les hommages du groupe, est soustrait à la vue (inhumation), détruit par le feu (crémation) ou substitué par une effigie.

Les masques mortuaires font partie des pratiques funéraires dès la République. Des masques en plâtre ont été retrouvés directement moulés sur le visage d'enfants. Les grandes familles aristocratiques portent ces imagines majorum en procession pour démontrer l'ancienneté et le pouvoir de la famille en deuil. (art. cit. p. 5).

Il est frappant de comparer les enfants morts de maladie ou d'accident aux nombreux cas d'infanticides [13]. Les enfants morts par la volonté de leurs parents sont littéralement des nonêtres pour lesquels ne se pose pas la question du crime, de la culpabilité et encore moins des rites. Pourtant l'ancienne Rome, craint les «malemorts » : la mort violente ou prématurée, les femmes mortes enceintes et la mort des très jeunes enfants. Par peur et pour ne pas provoquer de maladie, ces pourvoyeurs de « malemorts » sont donc exclus des nécropoles ou déposés à la limite des aires funéraires [10]. On ne garde jamais les cendres de ces enfants à la maison, contrairement à celles des proches.

Comment les enfants accomplissent-ils alors le «voyage » vers l'au-delà ? Les nouveaux-nés sont enterrés emmaillotés dans les vêtements qu'ils portaient pendant les quarante à soixante premiers jours de leur brève vie [20]. Les vêtements des plus grands reflètent leur statut, genre, ethnie et citoyenneté.

Les enfants des citoyens portent un collier avec des amulettes apotropaïques (protectrices) ou une bulla, médaillon en forme de disque agissant comme un dispositif de protection et un symbole de la citoyenneté romaine pour les garçons prépubères (ibid, 2012).

\section{L'avènement du christianisme et le rôle du baptême}

Le christianisme, qui devient religion d'État dans l'Empire romain au $\mathrm{IV}^{\mathrm{e}}$ siècle, puis le fondement de la société médiévale, bouleverse profondément la vision de l'au-delà et la signification de la mort. Dans une société globalement chrétienne (les juifs sont présents partout en Europe autour du $\mathrm{X}^{\mathrm{e}}$ et les musulmans principalement en Espagne) dans laquelle la religion conditionne la vie de la femme et de l'homme, de sa naissance à sa mort, le salut et le baptême deviennent essentiels. Si l'homme doit effacer les péchés commis au cours de sa vie, le baptême est la condition sine qua non pour être lavé du péché originel, échapper à la damnation éternelle et atteindre la vision béatifique. La mort d'un tout-petit non baptisé se trouve donc, dès les premiers siècles du christianisme, au cœur des débats qui aboutissent généralement à la condamnation des enfants morts sans baptême. Le concile de Carthage en 393 ap. J.-C. crée un grand changement : il interdit de baptiser les morts. Pour Saint-Augustin ( $\mathrm{V}^{\mathrm{e}}$ siècle ap. J.- C.) l'enfant n'est nullement immunisé contre le péché ; au contraire, le tout-petit porte le péché originel et s'il n'est pas baptisé, il sera damné pour 1 'éternité.

La dynastie carolingienne à partir de Pépin le Bref puis de son fils Charlemagne (742-814), impose la position des Pères de l'Église pour se définir comme chrétienne. L'idée de Saint-Augustin, selon laquelle les enfants morts sans baptême sont damnés se diffuse. Or, le baptême est à l'époque une affaire d'adulte, même si les textes canoniques recommandent de baptiser l'enfant rapidement, dans les 3 ans qui suivent sa naissance. Ce Haut Moyen-Âge, à partir de 476 et jusqu'en l'an 1000 , épouse encore la tradition hippocratique de la division de l'enfance en trois étapes :

- infantia, de 0 à 7 ans ;

- pueritia, de 7-12 ans pour les filles et 7-14 ans pour les garçons ;

- adolescentia, pour les enfants de $12 / 14$ à 21 ans [21].

La mortalité infantile de l'époque est à son sommet : en moyenne et jusqu'au début du $\mathrm{XX}^{\mathrm{e}}$ siècle, de 30 à $50 \%$ des enfants âgés de moins d'un an meurent à la naissance ou dans les jours qui suivent [22-25].

Lors de la mort d'un enfant, l'Église reconnaît et accepte les manifestations du deuil qui doivent toutefois rester modérées. Les parents ne doivent pas se montrer désespérés mais soulagés parce que, grâce au baptême, les enfants peuvent atteindre le ciel et jouir du bonheur éternel. La mort de l'enfant est considérée comme la volonté de Dieu, un acte voulu par lui, non gouvernable par des puissances terrestres et étranger à la responsabilité parentale. La seule responsabilité des parents n'est donc pas la santé de l'enfant vivant, mais bien son devenir au-delà de la mort : ils doivent seulement s'assurer que son âme atteigne la paix et le paradis. Le baptême est si essentiel pour le salut de l'âme qu'en 755, lors du concile de Ver, l'Église décide qu'en cas d'urgence, n'importe quel prêtre peut désormais baptiser un enfant où qu'il se trouve. Le sacrement du baptême n'est donc plus réservé à l'évêque, en visite occasionnelle dans la communauté pour y célébrer quelques cérémonies [26]. Il est indiqué en outre, dans les pénitentiels entre le $\mathrm{VII}^{\mathrm{e}}$ et le $\mathrm{X}^{\mathrm{e}}$ siècle (textes traditionnels destinés au clergé pour l'administration des pénitences), que la négligence des parents qui n'ont pas appelé le prêtre à temps, est passible d'un à sept ans de pénitence. Le prêtre, arrivé trop tard, est lui-même puni au jour du jugement dernier. La religion catholique insiste particulièrement sur cette culpabilité qui poursuit encore les parents jusqu'à aujourd'hui. C'est donc pour assurer le salut de leur enfant que les parents veulent apprendre les formules du baptême, en latin ou en langue vernaculaire, pour les réciter en cas de besoin [25].

Afin de mettre fin à ces pratiques et pour répondre à la peur des parents de voir l'âme de leur enfant séjourner en enfer, l'Église institue au XII ${ }^{\mathrm{e}}$ siècle, le baptême le plus proche de la 
naissance, soit le lendemain, sauf pour les familles aristocratiques qui doivent organiser une grande célébration [21].

On observe alors une apparition des émotions dans les sources historiques avec la reconnaissance de l'amour parental : la mort est maintenant présentée comme un drame et les larmes sont importantes dans les limites cadrées par l'Église.

Les baptêmes de l'époque sont collectifs : les enfants sont immergés dans des cuves, deux fois par an, à Pâques et à la Pentecôte [24].

Alors qu'à l'époque mérovingienne les sépultures d'enfants sont parfois isolées du cimetière et restent plus ou moins proches des habitations, beaucoup d'enfants sont enterrés, à partir de l'époque carolingienne, dans le cimetière, sous la gouttière, pour bénéficier de l'eau purificatrice qui descend du toit de l'église (près du mur sud). Parfois, le lieu d'inhumation se trouve le long des murs du cimetière.

L'Église définit un lieu pour inhumer les morts et un rituel pour accompagner les défunts aux $\mathrm{X}^{\mathrm{e}}-\mathrm{XI}^{\mathrm{e}}$ siècles [27]. Le cimetière devient un espace sacré, potentiellement profané par la présence de défunts non-chrétiens. Or, si les enfants baptisés y trouvent leur place et y sont inhumés comme les autres défunts, en revanche, les enfants non baptisés en sont toujours exclus [28].

Avant l'inhumation, le petit corps est placé dans la maison ou dans la cour pour la nuit, couché, recouvert d'un drap et entouré de cierges pour la veillée funèbre. Le glas sonne afin d'annoncer la nouvelle du rappel à Dieu de l'enfant. La communauté se joint aux prières des parents [22].

À partir du XIe siècle, sauver l'âme de l'enfant et lui permettre d'atteindre le paradis devient si essentiel que les techniques chirurgicales et gynécologiques se développent d'abord pour améliorer ses possibilités de salut physique et spirituel.

\section{L'ondoiement et les aménagements du baptême}

Puisqu'après le concile de Carthage de 393, le baptême des enfants morts a été totalement interdit par l'Église [22,29-32], on pratique, depuis le $\mathrm{XI}^{\mathrm{e}}$ siècle (et cela, jusqu'au $\mathrm{XIX}^{\mathrm{e}}$ ), l'ondoiement. C'est un baptême rapide, opéré d'urgence en cas de risque de décès de l'enfant. Il peut être suivi du rite complet et officiel du baptême, dans les rares cas où l'enfant survit. En effet, la sage-femme ou matrone, tente de faire couler de l'eau bénite sur la tête du fotus, seule partie noble, au travers de canules. Les parents sont alors soulagés car ils ignorent si l'enfant est mort avant ou après l'ondoiement et peuvent enterrer le nouveau-né en terre consacrée [33].

On observe alors une généralisation du baptême des toutpetits, les rites de naissance sont modifiés et une certaine inflexion dans l'émotion est perceptible, laissant une place pour l'expérience affective [34].

L'invention par l'Église d'une géographie de l'au-delà plus complexe, à partir de la seconde moitié du XII ${ }^{\mathrm{e}}$ siècle, marque un tournant essentiel dans l'histoire des femmes et des hommes face à la mort en Occident. La création du purgatoire, ce lieu intermédiaire dans l'au-delà [35], donne en effet un espoir supplémentaire d'échapper à la damnation éternelle. À la même époque, parce que l'Église lutte contre les rites païens et veut redonner du courage aux parents inquiets du sort de leurs enfants morts sans baptême, elle leur attribue un lieu spécifique d'où ils n'accèdent certes pas à la vision béatifique et à la paix éternelle, mais où ils échappent aux souffrances de l'enfer : le limbe des enfants (Limbus puerorum). Le limbe est d'abord une invention théologique dont les fidèles doutent et qui est peu représentée dans l'iconographie médiévale [33].

Les premiers sanctuaires à répit, l'expression est de Pierre Saintyves [36], voient le jour au $\mathrm{XI}^{\mathrm{e}}$ siècle [37], alors que le limbe n'existe pas encore. Si l'enfant n'a pas été baptisé avant de mourir, les parents l'emmènent au sanctuaire pour y prier les saints et surtout la Vierge afin d'obtenir qu'un miracle ressuscite l'enfant et permette son baptême [25].

Après le XIII ${ }^{\mathrm{e}}$ siècle et malgré l'invention du Limbus puerorum, les sanctuaires à répit continuent de voir affluer les parents en attente du miracle. Ces sanctuaires sont très nombreux en Europe occidentale (France, Allemagne, Belgique, Suisse, Autriche, nord de l'Italie). Les sanctuaires de NotreDame de Beauvoir (Moustiers-Sainte-Marie dans les Alpes de Haute-Provence), de Notre-Dame de Vassivière (Auvergne) et Notre-Dame de Longeborgne (Bramois en Suisse) comptent parmi eux. Plus de 260 sont dénombrés en France au XVI [23,24,30,32,38,39]. Même si elles seront interdites par la Curie romaine en 1729 , ces pratiques ne cessent qu'au XIX ${ }^{\mathrm{e}}$ siècle [33].

Le pèlerinage vers le sanctuaire à répit est rarement l'affaire des mères, fatiguées par l'accouchement. Ce sont donc les pères, la famille, les voisins qui portent le corps de l'enfant au sanctuaire, le plus souvent immédiatement après la naissance, parfois après avoir déterré l'enfant des endroits non consacrés. Le deuil est donc celui des parents mais aussi de toute la communauté. La longue et fatigante route devient une forme de pénitence, d'expiation des péchés des parents, « punis » avec la naissance d'un enfant mort qui n'a pu bénéficier du baptême [40].

L'iconographie générale de l'enfant change : jusqu'aux $\mathrm{XII}^{\mathrm{e}}-\mathrm{XIII}{ }^{\mathrm{e}}$ siècles, les artistes représentent les enfants avec des traits d'adulte, mais avec une petite taille [38]. Le XIII ${ }^{\mathrm{e}}$ siècle donne lieu à une image plus réelle, proche de l'enfant-Jésus dans les bras de la Vierge ou à l'image d'un petit ange nu, le putto et chérubin, symbole de l'âme [21,38,41].

L'enfant, également surnommé poupart, est malgré tout, souvent représenté comme un poupon, une poupée que l'on aime câliner ou mignoter.

Le XIV ${ }^{\mathrm{e}}$ siècle marque l'apparition des premières tombes représentant des enfants, seuls ou avec leurs parents. L'âge du petit défunt, la date de sa mort et le lien avec ses parents, sont inscrits sur les tombes familiales des élites. Il faudra attendre le $\mathrm{XV}^{\mathrm{e}}$ siècle pour que les familles de haut rang reconnaissent dans les épitaphes sur les tombes, les qualités de leurs enfants et regrettent que leur vie ait pris fin si tôt.

À partir de 1517, avec la publication des 95 thèses de Martin Luther et le début de la Réforme protestante, la situation religieuse, politique et sociale de l'Europe chrétienne subit une profonde métamorphose. Pour le protestantisme, la lecture de la Bible reste la seule voie à considérer et à suivre. La relation entre les fidèles et Dieu est directe, sans intervention de l'Église. Par conséquent, le péché doit être jugé par le 
Seigneur et seulement par lui. Les seuls sacrements autorisés sont le baptême et l'eucharistie, considérés comme les rites originaux. Contrairement aux catholiques toutefois, les enfants des protestants morts sans baptême, appartiennent tout de même au peuple des élus [33]. En effet, les partisans de la Réforme diffèrent des catholiques sur plusieurs point : le choix du baptême doit être conscient chez les adultes (pour les anabaptistes) alors que d'autres groupes protestants continuent de baptiser les plus petits sans qu'ils en aient bien évidemment formulé la demande. De plus, la miséricorde divine est accordée aux enfants non baptisés. Pour les protestants, Dieu décide de sauver les hommes, on ne peut donc pas « acheter» son salut comme chez les catholiques au Moyen-Âge.

L'Église anglicane, née du refus du roi Henry VIII de se soumettre à l'Église romaine et influencée par la pensée protestante, souligne que le sacrement du baptême n'est pas « absolument» mais seulement nécessaire au salut de l'enfant (elle sera suivie par la Suisse calviniste, les Pays-Bas, la Suède, la Norvège, la Finlande, le Danemark, les Balkans, l'Islande, etc.). Les chrisom children sont admis au paradis, bien que morts sans baptême. Initialement, le mot chrisom désigne le chrême versé sur la tête de l'enfant lors du baptême. Puis, par métonymie, il désigne le tissu destiné à protéger la tête de l'enfant, lors du versement de l'huile sainte et enfin l'ensemble de son vêtement (chrisom-cloth). Jusqu'au début du $\mathrm{XVI}^{\mathrm{e}}$ siècle, le mot permet de distinguer un nouveau-né de moins d'un mois après son baptême. À la fin du $\mathrm{XVI}^{\mathrm{e}}$ et jusqu'à la seconde moitié du $\mathrm{XVII}^{\mathrm{e}}$ siècle, le terme de Chrisom child est également utilisé pour désigner les enfants morts non baptisés[39].

Dans le cadre de la Contre-Réforme, et pour répondre aux positions des protestants, le concile de Trente en 1563 rappelle que la naissance, comme la mort éventuelle de l'enfant, sont voulues par Dieu et que seul le baptême permet l'accès au paradis, y compris pour le tout-petit.

\section{L'âge baroque et la diffusion des pratiques de deuil dans les milieux modestes}

Selon Vovelle, le rituel du deuil est plutôt manifesté par les élites car cette forme d'expression sophistiquée complique les émotions. Ce processus d'autocontrainte se diffuse progressivement dans toutes les sociétés européennes [42]. Le XVII ${ }^{\mathrm{e}}$ siècle voit la mortalité infantile diminuer légèrement même si 30 à $50 \%$ des enfants meurent avant leur première année de vie. Les cimetières se modifient peu à peu. À la fin de l'Ancien Régime, en plus d'un portrait sur le tombeau du défunt, déposer les corps des membres de la même famille dans une chapelle différenciée à l'intérieur du cimetière se généralise bien que cette pratique exista déjà depuis le Moyen-Âge [38].

À partie du XVIII ${ }^{\mathrm{e}}$ siècle, les églises perdent leur statut de lieu d'inhumation privilégié. Pour des raisons d'hygiène et d'espace, le cimetière devient le lieu funéraire officiel [43]. Un emplacement est entièrement réservé aux enfants baptisés. En revanche, ceux qui n'ont pas reçu le baptême ne peuvent toujours pas être enterrés en terre consacrée par peur de la souiller [30-33].

Cet « avantage » réservé aux baptisés est mis en avant par l'Église pour limiter les émotions tristes des parents, intégrer l'enfant à la communauté et redonner un statut important au « petit intercesseur ».

\section{Conclusion}

Cette première partie a abordé une période dans laquelle la mortalité périnatale et infantile est très élevée et où le deuil périnatal est une réalité omniprésente. Les sources démontrent que les parents fortunés regrettent la mort de leur enfant, tandis que l'insuffisance de preuves ne nous permet pas de conclure pour les plus pauvres. L'imprégnation religieuse des sociétés antique et médiévale permet d'avoir accès aux connaissances sur le deuil et plus précisément sur le devenir des enfants morts. La puissance du christianisme prend appui sur le baptême, nécessité absolue pour le salut de l'enfant. Cette obligation engendre une course au baptême avant la mort et même des tentatives fictives de « remise en vie » le temps du sacrement dans les sanctuaires à répit.

Les travaux sur l'Histoire des émotions nous apportent de nouveaux éléments pour penser les expériences émotionnelles de nos ancêtres. Au-delà des révolutions des mouvements religieux comme le protestantisme, le développement des sciences, et particulièrement de la médecine $\mathrm{a}$, à partir des Lumières, une influence majeure. Les études sur le corps humain et l'avènement d'une vision positiviste de l'Homme remettent en effet en question la symbolique de la mort de la créature divine qui perd en spiritualité et en singularité au sein de l'univers.

L'article suivant celui-ci traite de l'évolution des pratiques et rites funéraires à la mort d'un jeune enfant à partir de la naissance, des Lumières jusqu'à nos jours. Sa conclusion portera sur l'ensemble de la période étudiée : de la Préhistoire jusqu'au début du $\mathrm{XXI}^{\mathrm{e}}$ siècle.

\section{Déclaration de liens d'intérêts}

Les auteurs déclarent ne pas avoir de liens d'intérêts.

\section{Références}

[1] Thomas L-V. Anthropologie de la mort. Paris: Payot; 1988, 538 p. [Bibliothèque scientifique Payot].

[2] Van Gennep A. Les rites de passage : étude systématique des rites de la porte et du seuil, de l'hospitalité, de l'adoption, de la grossesse et de l'accouchement, de la naissance, de l'enfance. Paris: Picard; 2011.

[3] Bacqué M-F. Apprivoiser la mort : psychologie du deuil et de la perte. Paris: O. Jacob; 2002, 283 p.

[4] Bacqué M-F. Parler du deuil pour éviter de parler de la mort ? La société occidentale face aux changements démographiques et culturels du XXIe siècle. Ann Med Psychol Rev Psychiatr 2013;171(3):176-81.

[5] Mohen J-P. Les rites de l'au-delà. Paris: O. Jacob; 1995, 329 p.

[6] Corbin A, Courtine J-J, Vigarello G. Introduction générale. In: Corbin A, Courtine J-J, Vigarello G, editors. Histoire des émotions. Paris: Seuil; 2016. p. 5-11.

[7] Riel-Salvatore J, Clarke GA. Grave markers. Curr Anthropol 2001;42(4):449-79.

[8] Maureille B. Les premières sépultures. [Éd. mise à jour]. Paris: Éd. le Pommier Cité des sciences et de l'industrie [Universcience]; 2013, 133 p. [Le collège].

[9] Tillier A-M. La mort des périnatals et nourrissons en Préhistoire. In: Coste M-C, Alduc-Le Bagousse A, editors. Journée d'étude sur les pratiques 
funéraires autour de l'enfant mort au Moyen-Âge Blandy-les-Tours, le 14 novembre 2009. Milan: Silvana Editoriale; 2011.

[10] Durand R. Données paléodémographiques et classes d'âge immatures : recrutement et gestion des enfants dans les espaces funéraires galloromains. In: Nasciturus, infans, puerulus vobis mater terra : la muerte en la infancia. Servei d'Investigacions Arqueològiques i Prehistòriques; 2008. p. 41-56.

[11] Hermary A, Dubois C.Maison Méditerranéenne des Sciences de l'Homme éditeurs. Arles: Éd. Errance; 2012, 460 p. [L’ enfant et la mort dans l'antiquité].

[12] Frank C. L'inhumation des enfants en Mésopotamie à l'Âge du Bronze. In: Nasciturus, infans, puerulus vobis mater terra: la muerte en la infancia. Servei d'Investigacions Arqueològiques i Prehistòriques; 2008. p. 231-56.

[13] Baills-Talbi N, Blanchard P. Sépultures de nouveau-nés et de nourrissons du 1er âge du Fer au haut Moyen Âge découvertes hors des contextes funéraires traditionnels sur les territoires carnute, turon et biturige cube : inventaire, synthèse et interprétations. Suppl Rev Archeol Cent Fr 2006;29(1):157-205.

[14] Sartre M. Les Grecs. In: Corbin A, Courtine J-J, Vigarello G, editors. Histoire des émotions. Paris: Seuil; 2016. p. 17-63.

[15] Delcourt M. Stérilités mystérieuses et naissances maléfiques dans l'antiquité classique [Internet]. Liége: Presses universitaires de Liège; 1986 [cité 9 sept 2017]. Disponible sur : http://books.openedition.org/pulg/647.

[16] Jobbé-Duval E. Les morts malfaisants (larvae, lemures) : d'après le droit et les croyances populaires des Romains. Chambéry: Exergue; 1924.

[17] Dasen V. Archéologie funéraire et histoire de l'enfance dans l'Antiquité : nouveaux enjeux, nouvelles perspectives. In: L'enfant et la mort dans l'Antiquité I Nouvelles recherches dans les nécropoles grecques Le signalement des tombes d'enfants. Paris: De Boccard; 2010. p. 19-44 [Travaux de la Maison Archéologie \& Éthnologie].

[18] Dasen V. La mort des enfants à Rome : l'impossible chagrin ? Fribourg U de, éditeur, 15. Vouivre Cah Romands Psychol Anal; 2006. p. 29-37.

[19] King M. Commemoration of Infants on Roman Funerary Inscriptions. In: Oliver GJ, editor. The Epigraphy of Death: Studies in the History and Society of Greece and Rome. Liverpool University Press; 2000. p. $117-54$

[20] Carroll M. The Roman child clothed in death. In: Dressing the dead in classical antiquity. Stroud: Amberley; 2012. p. 134-47.

[21] Heywood C. A history of childhood: children and childhood in the West from medieval to modern times. Cambridge, UK ; Malden, Mass: Polity Press; 2001, $231 \mathrm{p}$.

[22] Morel M-F, Lett D. Une histoire de l'allaitement. Paris: de la Martinière; $2006,160 \mathrm{p}$.

[23] Einaudi-de Siano M-A. Le décès périnatal : vécu parental comprendre, décrire, améliorer. Université de la Méditerranée Aix-Marseille II; 2008. p. 98.

[24] Alexandre-Bidon D, Lett D. Les Enfants au Moyen Age : Ve-XVe siècles. Paris: Hachette; 1997, 280 p. [La Vie quotidienne].

[25] Lett D. L'enfant des miracles : enfance et société au Moyen Age (XIIe-XIIIe siècle). Paris: Aubier; 1997, 396 p. [Collection historique].
[26] Treffort C. L'église carolingienne et la mort : christianisme, rites funéraires et pratiques commémoratives. Lyon: Centre interuniversitaire d'histoire et d'archéologie médiévales: Presses universitaires de Lyon; 1996, 216 p. [Collection d'histoire et d'archéologie médiévales].

[27] Lauwers M. Naissance du cimetière : lieux sacrés et terre des morts dans l'occident médiéval. Paris: Aubier; 2005, 393 p. [Collection historique].

[28] Coste M-C, Alduc-Le Bagousse A.Journée d'Étude sur les Pratiques Funéraires Autour de l'Enfant Mort au Moyen Âge, éditeurs. Milan: Silvana Ed. [u.a.]; 2011, 135 p.

[29] Treffort C. Mémoires carolingiennes : l'épitaphe entre célébration mémorielle, genre littéraire et manifeste politique ; (milieu VIIIe - début XIe siècle). Rennes: Presses Univ. de Rennes; 2007, 383 p. [Histoire].

[30] Morel M-F. Images du petit enfant mort dans l'histoire. Études Sur Mort 2001;119(1):17-38.

[31] Ariès P. L'homme devant la mort. Paris: Éditions du Seuil; 1977, 641 p. [Univers historique].

[32] Tzortzis S, Séguy I. Pratiques funéraires en lien avec les décès des nouveaux-nés. Socio-Anthropol 2008;(22):75-92.

[33] Gélis J. Les enfants des limbes : mort-nés et parents dans l'Europe chrétienne. Paris: Audibert; 2006, 396 p.

[34] Boquet D, Nagy P. Une histoire des émotions incarnées. Mediev Lang Textes Hist 2011;61(61):5-24.

[35] Le Goff J. La naissance du Purgatoire. Paris: Gallimard; 1981, 509 p. [Bibliothèque des histoires].

[36] Saintyves P. Les résurrections d'enfants mort-nés et les sanctuaires à répit. Rev Ethnogr Sociol 1911;2:65-74.

[37] Delattre V. Les sépultures de nouveaux-nés au Moyen-Âge : l'hypothèse d'un sanctuaire à répit précoce à Blandy-Les-Tours. France: Seine-etMarne. In Servei d'Investigacions Arqueològiques i Prehistòriques; 2008. p. $183-210$.

[38] Ariès P. L' enfant et la vie familiale sous l'Ancien régime : avec 26 illustrations hors texte. Nouvelle éd. Paris: Éd. du Seuil; 1973, 501 p. [L' univers historique].

[39] Coster W. Tokens of innocence: infant baptism, death and burial in early modern England. In: Gordon B, Marshall P, editors. The place of the dead: death and remembrance in late medieval and early modern Europe. Cambridge, UK; New York: Cambridge University Press; 2000. p. 266-87.

[40] Lett D. Comment faire le deuil d'un enfant mort à la fin du Moyen Âge. In: Hanus M, Baron E, De Broca A, editors. La mort d'un enfant Fin de vie de l'enfant, le deuil des proches. Paris: Vuibert; 2005. p. 19-26.

[41] Oosterwijk S. The medieval child: an unknown phenomenon? In: Harris SJ, Grigsby BL, editors. Misconceptions about the Middle Ages. New York: Routledge; 2008. p. 230-5.

[42] Vovelle M. La mort et l'Occident : de 1300 à nos jours ; précédé de La mort, état des lieux. Nouv. éd. Paris: Gallimard; 2000, 793 p. [Bibliothèque illustrée des histoires].

[43] Bertrand R, Carol A, editors. Aux origines des cimetières contemporains : les réformes funéraires de l'Europe occidentale XVIIIe-XIXe siècle. Aixen-Provence: Presses universitaires de Provence; 2016, 377 p. [Corps \& ames]. 